\title{
Optical characterisation of a camera module developed for ultra-low NEP TES detector arrays at FIR wavelengths
}

D. Morozov, R. Sudiwala, P. A. R. Ade, D. J. Goldie, D. M. Glowacka, et al.

D. Morozov, R. Sudiwala, P. A. R. Ade, D. J. Goldie, D. M. Glowacka, S. Withington, N. A. Trappe, "Optical characterisation of a camera module developed for ultra-low NEP TES detector arrays at FIR wavelengths," Proc. SPIE 9914, Millimeter, Submillimeter, and Far-Infrared Detectors and Instrumentation for Astronomy VIII, 991409 (19 July 2016); doi: $10.1117 / 12.2232139$

Event: SPIE Astronomical Telescopes + Instrumentation, 2016, Edinburgh, United Kingdom 


\title{
Optical characterisation of a camera module developed for ultra-low NEP TES detector arrays at FIR wavelengths
}

\author{
D. Morozov**a , R. Sudiwala ${ }^{\mathrm{a}}$, P. A. R. Ade ${ }^{\mathrm{a}}$, D. J. Goldie ${ }^{\mathrm{b}}$, D. M. Glowacka ${ }^{\mathrm{b}}$, S. Withington ${ }^{\mathrm{b}}$, N. A. \\ Trappe ${ }^{\mathrm{C}}$

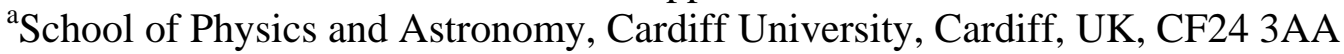 \\ ${ }^{\mathrm{b}}$ Quantum Sensors Group, Cavendish Laboratory, University of Cambridge, JJ Thompson Av., \\ Cambridge, UK, CB3 OHE \\ 'Department of Experimental Physics, National University of Ireland, Maynooth, Ireland
}

\begin{abstract}
Here we report on the optical design and on the spectral-spatial characterisation of a small 16 pixel camera. The prototype uses TES detectors with NEPs $\sim 10^{-16} \mathrm{~W} / \mathrm{Hz}^{0.5}$ which have been fabricated with near identical optical coupling structures to mimic their much lower NEP counterparts $\left(\sim 10^{-19} \mathrm{~W} / \mathrm{Hz}^{0.5}\right)$. This modification, which is achieved through changing only the pixel thermal conductance, $G$, has allowed us to perform spectral/spatial cryogenic testing using a $100 \mathrm{mK}$ ADR to view room temperature thermal sources. The measurements show a flat spectral response across the waveband and minimal side lobe structure in the antenna patterns down to $30 \mathrm{~dB}$.
\end{abstract}

Keywords: SPICA, SAFARI, TES, Optical Coupling in Far Infrared, Profiled wall pyramidal horns

* Dmitry.Morozov@astro.cf.ac.uk

\section{INTRODUCTION}

SAFARI is a proposed FIR instrument for SPICA space based observatory with $2.5 \mathrm{~m}$ cooled $(<8 \mathrm{~K})$ primary mirror. SAFARI consists of a spectrometer covering the wavelengths from $34-210 \mu \mathrm{m}$ with science goals of understanding of planetary system formation, the life cycle of dust and gas in the galaxy and the formation of galaxies. The SAFARI wavelength coverage is divided into 3 bands in the focal plane covering the wavelength ranges from 35-60 $\mu \mathrm{m}$ (S-band), 60-110 $\mu \mathrm{m}$ (M-band), and 110- $210 \mu \mathrm{m}$ (L-band). In order to take advantage of the reduction in background power provided by the cooled telescope, SAFARI requires detectors with a combination of low optical NEP $\left(\sim 2 \times 10^{-19}\right.$ $\mathrm{W} / \mathrm{Hz}^{0.5}$ ) and high optical efficiency [1,2].

Transition Edge Sensors (TES) are the baseline detectors for the SAFARI instrument. Coupled with circular and pyramidal horns, L- and S-band TES detectors have demonstrated high optical efficiency and low NEP values (few $10^{-19}$ $\mathrm{W} / \mathrm{Hz}^{0.5}$ ) [3-5]. Nevertheless, improvement and better optical characterization of the optical coupling is still needed. Current work is aimed at further study and enhancement of a horn coupled TES camera. Here we describe the design of the camera module, optical components and TES detectors for the SAFARI L-band. We also report on the optical measurements of the TES pixels in $4 \times 4$ prototype camera, which were designed to handle the room temperature background and at the same time having the same optical coupling arrangement as low-background TES detectors.

\section{DESIGN OF THE CAMERA MODULE AND OPTICAL SETUP}

\subsection{Camera module}

Here we propose a unique camera architecture for future FIR low background instruments using ultra-low noise TES detectors. It consists of using profiled wall pyramidal horns in a square array feeding an array of TES detectors on a 
single silicon chip with an appropriate back-short. Important factors that drive this design are the need for a compact form with robust structure and tight tolerances $<5 \mu \mathrm{m}$ which creates a Faraday cage around the detectors and guards against stray light. Figure 1 shows an exploded view of the detector focal plane assembly. The cold mounting plate and the detector mount plate form a fully enclosing Cu shield around the SQUID readout, which is on the back surface of the detector mount. The detector mount top plate and the detector plate fully enclose the ultra-sensitive detectors to shield them from electromagnetic fields and stray radiation except that coming through the prescribed optical path through the horn apertures. The horn block exit apertures lie close to the detector wafer ( $\sim 15 \mu \mathrm{m}$ vacuum gap) to ensure optimum optical coupling. A silicon wafer back-short plate is integrated with the detector wafer to provide a compact assembly

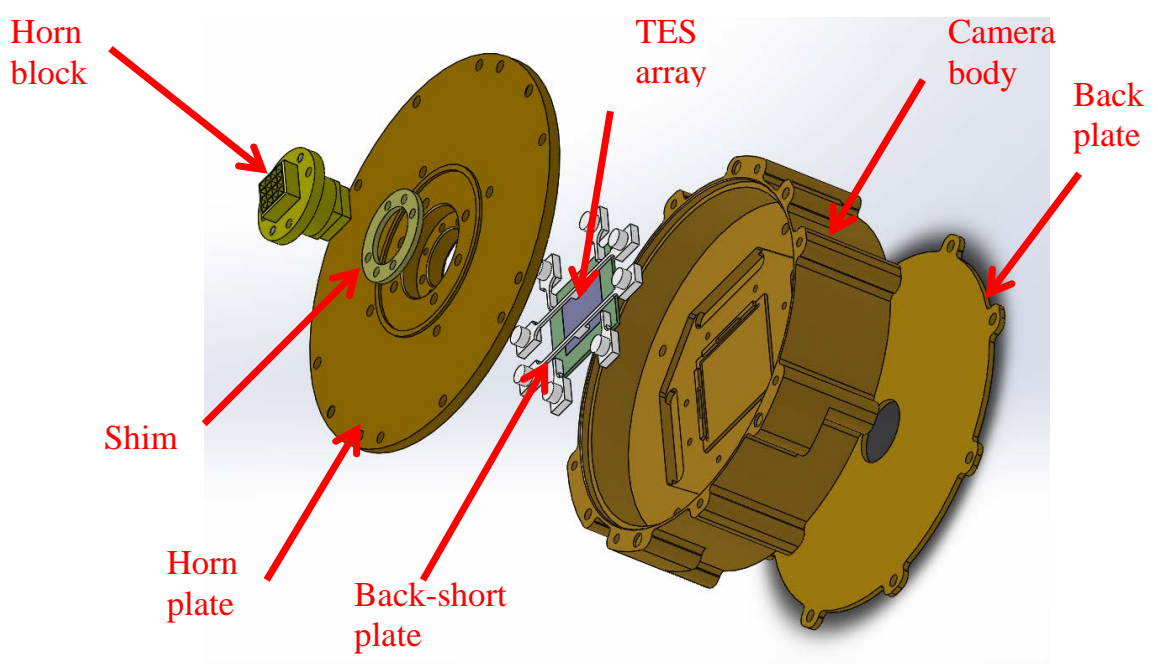

Figure 1: Exploded model view of the camera module.

that is held in place by the dielectric clamps shown. The integrated stack is designed to be correctly aligned only when cold so the mounting structure allows shrinkage of metal components with respect to the silicon wafer. The annular spacer shown between the horn plate and the detector mount top is used to adjust the gap between the horn exit apertures and the detector wafer.

We have manufactured the camera module and developed the integration procedure (using a non-contact surface profiler by Polytec [6]), which ensures the tight tolerances of air gaps crucial for the optical coupling.

\subsection{Horn design and manufacture}

The prototype $4 \times 4$ horn designs are based on a pyramidal few-mode horn array that can couple the detectors to the
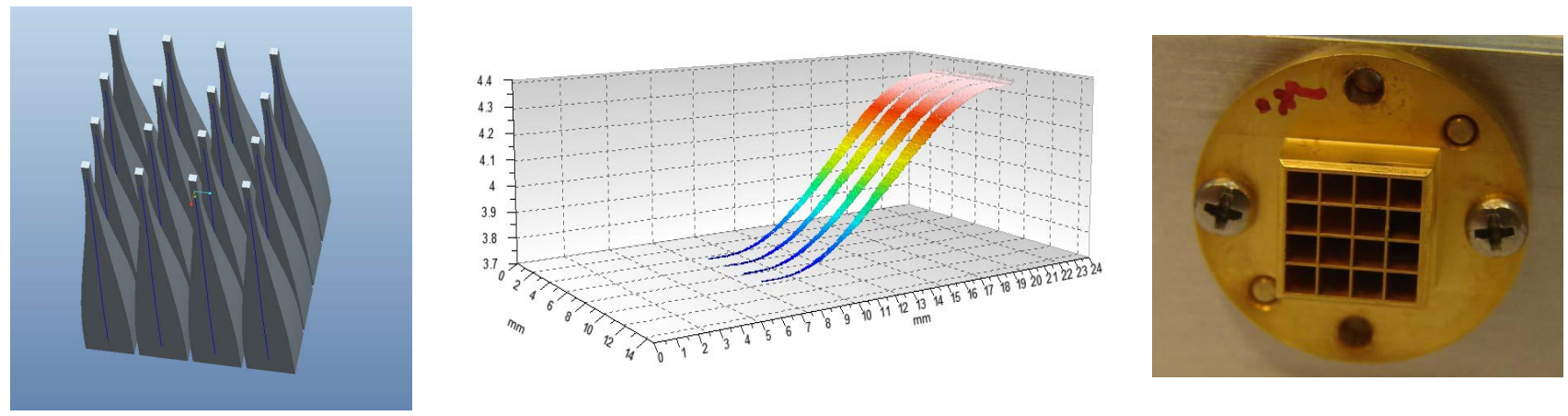

Figure 2: 3D CAD drawing of mandrel (left). Surface profile of the L-band mandrel (centre). Optical picture of the horn entrance apertures (right). 
instrument focal plane. It was expected that a typical FIR instrument would present an F-16 beam to the detector horn so emphasis was placed on the centre of the beam pattern out to $\sim 5$ degrees. The initial design study showed that a profiled pyramidal design could achieve high efficiency, good beam directivity whilst minimising the horn length. These horns are profiled with a $\sin ^{2}$ form to couple the detectors to the instrument's optics as efficiently as possible. L-band horns have an entrance aperture size of $1450 \mathrm{um}$ and exit aperture size of $235 \mathrm{um}$ and the total length of $13.527 \mathrm{~mm}$.

The horn manufacture process (performed by Thomas Keating [7]) is to electroform onto a wire eroded sacrificial aluminium mandrel. After electroforming the outer form is machined whilst the mandrel is intact to register the optical axis. Lastly, the aluminium is etched out to reveal the horn array. The main challenge is that any imperfections arising in the mandrel including surface roughness would transfer from the mandrel to the electroformed inner horn surfaces. These imperfections would become more apparent at the short wavelengths where the roughness scale is approaching the wavelength enhancing scattering losses. As part of the manufacturing process, the surface roughness and compliance with the $\sin ^{2}$ profile was monitored by non-contact surface profiler. Figure 2 shows 3D CAD model and the surface profile of the manufactured aluminium mandrel. Entrance apertures of 4x4 horn block are also shown in Figure 2.

Horn performance was first extensively modelled using pyramidal SCATTER code to compute a multi-moded far field beam patterns and to optimize the parameters of $\sin ^{2}$ horn profiling. To verify the performance of manufactured horn blacks we have measured a spectral response and antenna beam patterns of horns connected in back-to-back configuration. We have observed an average measured L-band throughput for a single horn of $\sim 70 \%$. The measured width of antenna pattern is $\sim 14$ degrees and it is in line with the modelling expectation.

\subsection{Optical test setup}

Cryogenic test setup is based on Adiabatic Demagnetisation Refrigerator (ADR) cooled by liquid helium and nitrogen
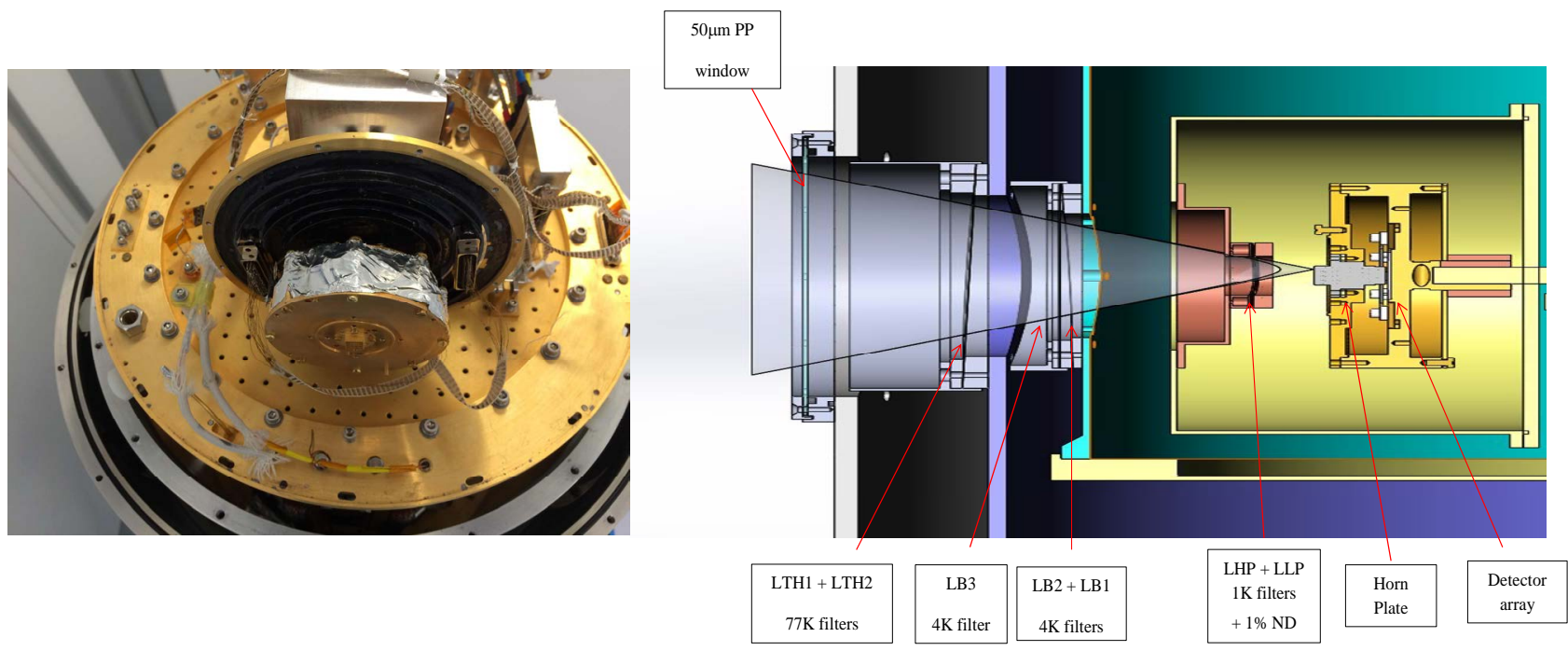

Figure 3: Image of the ADR with mounted camera module (left). CAD model of the ADR optical configuration (right).

supplied by Janis. The cold stage cools down to base temperature of $69 \mathrm{mK}$ without optical power loading. The temperature of the cold stage is monitored by an RuOx sensor, which is also used in a servo loop to maintain the stage at constant temperature by controlling the current applied to the ADR magnet. Figure 3 shows an image of the camera module mounted on the cold finger with vacuum and radiation shields removed. The ADR has a side looking optical window, which is aligned to the horizontal axis of the low temperature $(100 \mathrm{mK})$ stage. A large, $91 \mathrm{~mm}$ diameter, vacuum window allows full exploration of the antenna pattern of the subarray pyramidal horns using external radiation sources. Figure 3 shows a cut away view through the side looking optics showing the low temperature detector housing, the filter and shield locations and the vacuum window. For clarity the un-vignetted field of view extent of the camera is shown which is much larger than the design horn pattern width allowing the full horn antenna pattern to be explored.

A set of filters has been designed to have good in-band transmission along with good out of band rejection. The passband is defined by a high pass low pass combination. There are then 3 additional low pass blockers to give a total FIR rejection $\sim 10^{9}$. In addition two thermal filters are used for testing with external sources viewed through the cryostat 
window. These latter filters are only necessary for ground tests and would not be implemented in a flight instrument. The window is a $50 \mu \mathrm{m}$ PP film that has near $100 \%$ transmission at the centre of L- band. The losses and/or emission from this window are negligible. In this configuration, thermal filters are mounted on the $77 \mathrm{~K}$ shield and are crucial for maintaining LHe hold time of the ADR. Figure 4 shows the filter stack transmission spectra as well as spectra of the additional high frequency blocking filters used in the stack.

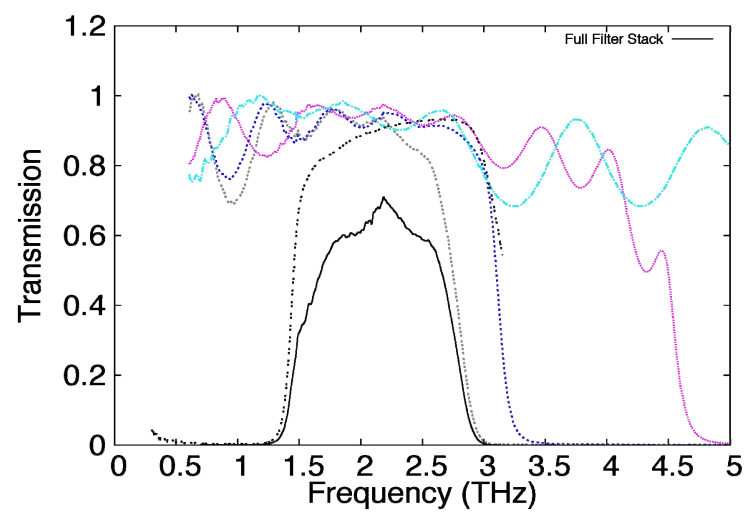

Figure 4: Transmission spectra for the band defining and blocking filters.

\subsection{TES arrays and readout}

TES detectors were designed with NEPs $\sim 10^{-16} \mathrm{~W} / \mathrm{Hz}^{0.5}$ with near identical optical coupling structures to mimic their much lower NEP counterparts ( $10^{-19} \mathrm{~W} / \mathrm{Hz}^{0.5}$ described in [8]). This modification, which is achieved through changing only the pixel thermal conductance, has allowed us to perform optical testing with room temperature thermal sources. Individual Detector consists of a $200 \mathrm{~nm}$-thick $\mathrm{Si}_{X} \mathrm{~N}_{y}$ membrane supporting a Mo/Au thermometer with $T_{\mathrm{C}} \sim 600 \mathrm{mK}$ and layer thicknesses of 40/50 $\mathrm{nm}$. A Ta absorber is situated next to the Mo/Au thermometer. The absorber layer has surface resistance of $440 \mathrm{Ohm} /$ square to match free space impedance. The entire structure is suspended on four $\mathrm{Si}_{x} \mathrm{~N}_{y}$ beams, which thermally decouples the absorber and thermometer from the thermal bath. Niobium wiring is used to contact the thermometer and absorber. The $4 \times 4$ arrays were fabricated with pixels having filled (un-patterned) and meshed absorbers with sizes of 320x320 um ${ }^{2}$ and 250x250 um². Figure 5 shows an image of 4x4 array A3 and magnified pixel TES_1 with meshed absorber.

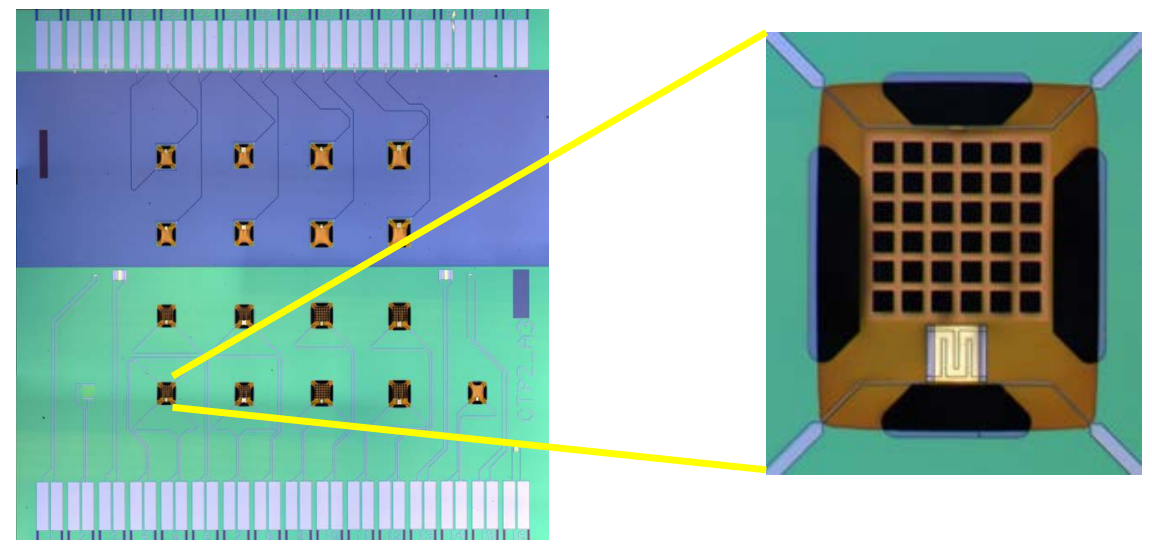

Figure 5: Image of the array A3 (left). Close-up image of the pixel TES 1 with meshed absorber (right).

In this study we have investigated the optical performance of TES pixels from 2 arrays: array A3 (3 meshed pixels) and array A5 (2 meshed and 2 filled pixels). Throughout the paper the pixels are identified as follows: TES_1 (meshed 250 
$\mu \mathrm{m}$ absorber), TES_5 (meshed $250 \mu \mathrm{m}$ absorber), TES_10 (meshed $320 \mu \mathrm{m}$ absorber) in Array A3; TES_1 (meshed 250 $\mu \mathrm{m}$ absorber), TES_5 (meshed $250 \mu \mathrm{m}$ absorber), TES_7 (filled $250 \mu \mathrm{m}$ absorber) and TES_16 (filled $320 \mu \mathrm{m}$ absorber).

Our read-out consists of 6 independent readout/bias channels. The schematic of the readout circuit is shown on Figure 6 where $R_{b}$ is bias resistor, $R_{S}$ is the shunt resistor, $V_{b}$ is the bias voltage and $V_{F B}$ is the voltage at the output of the SQUID current amplifier. The TES is shown as a variable resistor $\mathrm{R}_{\text {TES }}$ and $\mathrm{L}_{\text {in }}$ is the inductance of the input coil of the SQUID.
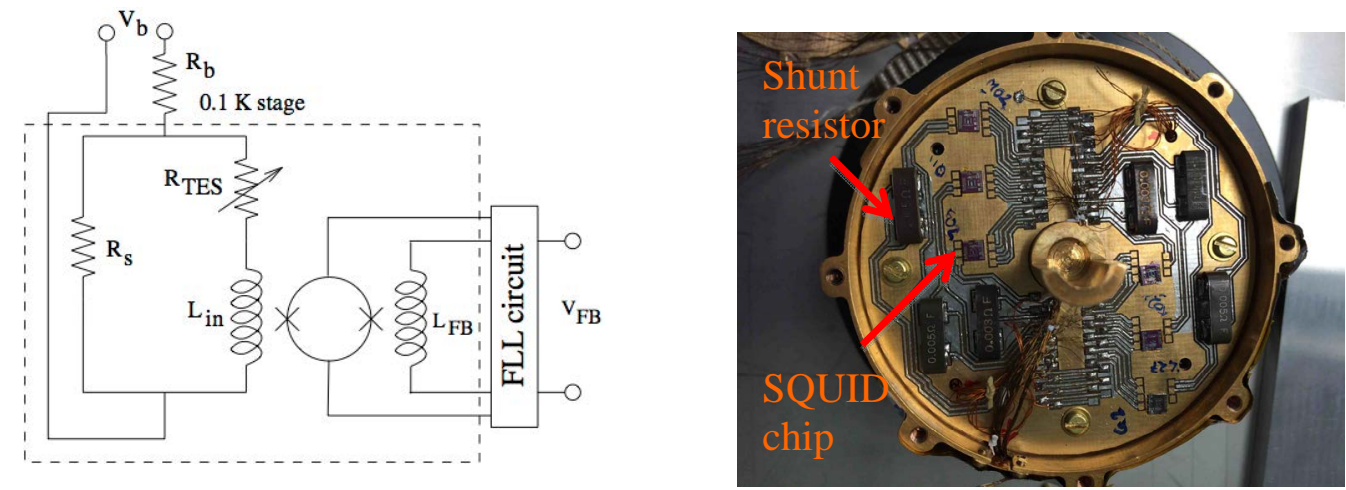

Figure 6: Readout circuit of TES detector (left) with SQUID operated in the Flux Locked Loop (FLL) mode. Image of the read-out board of the camera module (right).

We used PTB [9] SQUID chips and warm SQUID electronics supplied by Magnicon [10]. Figure 6 also shows an image of the read-out board on the reverse side of the detector mount. The shunt resistors and wire bonded SQUID chips can be seen. We used a set of shunt resistors with $\mathrm{R}_{\mathrm{s}}$ values of 3, 5 and $10 \mathrm{mOhm}$ and SQUIDs with $\mathrm{L}_{\text {in }}$ values of 65 and 150 $\mathrm{nH}$.

\section{RESULTS AND DISCUSSION}

\subsection{Dark measurements}

For verification purposes, pixels in the array A3 were first measured in a dark environment to obtain thermo-electrical parameters such as normal resistance $\left(R_{n}\right)$, thermal conductance $(G)$, critical temperature $\left(T_{c}\right)$ and to estimate the phonon fluctuation limited noise equivalent power $\left(\mathrm{NEP}_{\mathrm{ph}}\right)$. To perform dark measurements, optical apertures of the $100 \mathrm{mK}$ subarray assembly module, $1 \mathrm{~K}$ stage, $4.2 \mathrm{~K}$ stage and $77 \mathrm{~K}$ were blanked to ensure that there is no optical power absorbed by devices under the tests.

At equilibrium $P_{\mathrm{TES}}$ is described by the equation:

$$
P_{T E S}=k\left(T_{c}^{n}-T^{n}\right),
$$

where $T_{c}$ is the critical temperature, $n$ depends on the heat transfer mechanism and $k$ is a constant. The differential thermal conductance $G$ can be found using:

$$
G=\frac{d P}{d T}=n k T^{n-1}
$$

And the phonon noise limited noise equivalent power ( $\left.\mathrm{NEP}_{\mathrm{ph}}\right)$ of the TES is given by:

$$
N E P_{p h}=\sqrt{\gamma 4 k_{B} T^{2} G}
$$

where $\gamma$ is in the range form 0 to 1 . In this paper $\gamma$ is assumed to be 0.5 . 
Dissipated TES power $\left(\mathrm{P}_{\mathrm{TES}}\right)$ can be found from the IV curves taken in the range of the bath temperatures from $100 \mathrm{mK}$ to $600 \mathrm{mK}$. IV plots and $\mathrm{P}_{\text {TES }}$ plots for TES_1 from the array A3 are shown on Figure 7. For three measured pixels on the array A3 we performed a least square fitting to equation (1) and found that $T_{c}$ was in the range of $583-600 \mathrm{mK}, R_{n}=$ $134-208 \mathrm{mOhm}, \mathrm{G}=1.48-2.71 \mathrm{nW} / \mathrm{K}$ and $\mathrm{n}=3.14-3.17$. Using equation (3) we have estimated the phonon fluctuation noise from the dark measurements, which is in the limits of $6.06-8.21 \times 10^{-17} \mathrm{~W} / \mathrm{Hz}^{0.5}$ for three measured pixels.
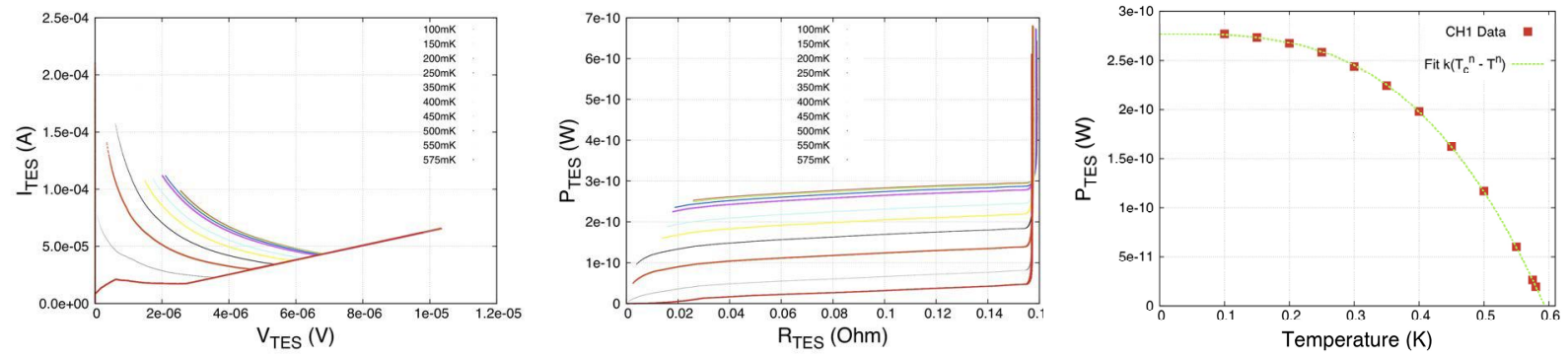

Figure 7: Data for the pixel TES_1. Left: IV curves, centre: $\mathbf{P}_{\text {TES }}$, right: $\mathbf{P}_{\text {TES }}$ vs. bath temperature, points show the data and curve is a fit to equation (1).

\subsection{Optical measurements: spatial and spectral response.}

To perform the optical measurements, a horn block was installed in front of the TES array. The ADR filter configurations for both arrays were identical with the exception that an additional neutral density filter was used to prevent saturation for sub-array A5. The set of optical tests consisted of the (i) beam pattern measurement, (ii) spectral response and (iii) optical efficiency measurement using the hot (300K) and cold (77K) loads.

To map the beam we rotated a mercury lamp source about the entrance apertures of the horn block and measured the

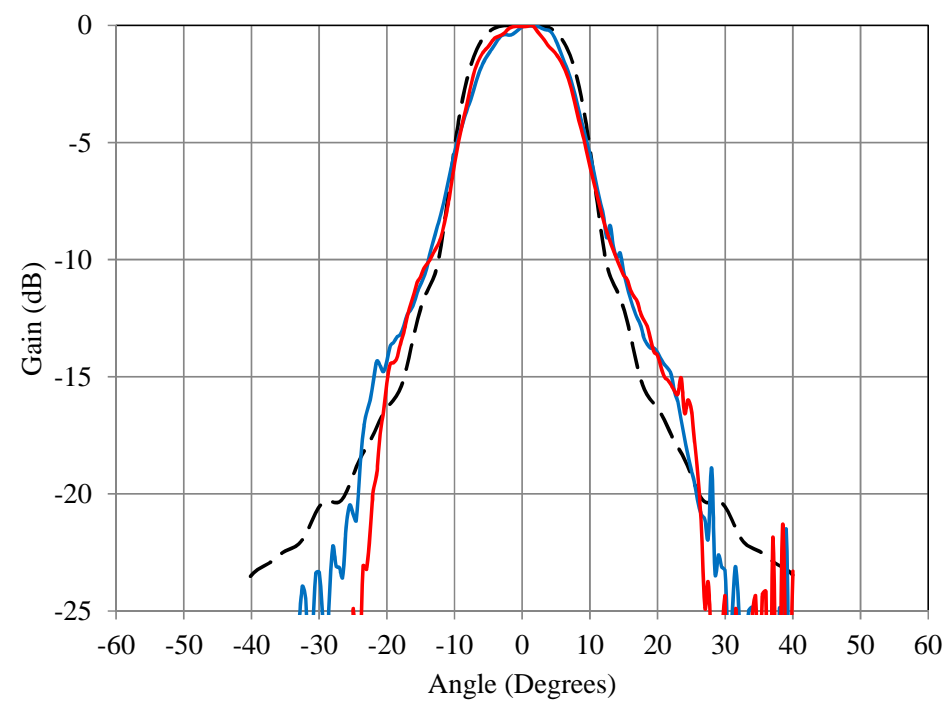

Figure 8: Camera antenna patterns of pixel TES_7 (blue curve) with filled absorber and meshed absorber pixel TES_5 (red curve). Both pixels are in the array A5. Dashed curve is the averaged model expectation profile for L-band horn.

TES signal at each angular step. Beam patterns were measured with arrays held at base temperature $T=300 \mathrm{mK}$. Pixels TES_1, TES_5 and TES_10 in array A3 were biased with $\mathrm{V}_{\mathrm{b}}=0.51 \mathrm{~V}$. Pixels TES_16 and TES_7 in Array A5 were biased with $\mathrm{V}_{\mathrm{b}}=0.63 \mathrm{~V}$; pixels TES_5 and TES_1 in Array A5 were biased with $\mathrm{V}_{\mathrm{b}}=0.706 \mathrm{~V}$. The beam patterns are shown in Figure 8 for both the meshed and filled absorber types. The plots have been normalised to remove gain 
(responsivity) differences between the pixels. The signal ratio of the central peak to the noise level is more than $20 \mathrm{~dB}$. A comparison of these measured profiles with the averaged model prediction (averaged of profiles simulated for 110, 160 and 210 um radiation) is shown in Figure 8. Pixels with meshed absorbers have more symmetrical profiles than pixels with filled absorbers. This comparison was done for the pixels in array A5, where measurements were performed in the same run to avoid uncertainties of alignment introduced by changing the arrays in the camera.

Spectra were measured with Martin -Puplett polarizing Fourier Transform Spectrometer (FTS). TES pixels were held at base temperature $300 \mathrm{mK}$ and biased with the same corresponding voltages as for beam pattern measurements. FTS setup schematics is shown on Figure 9 (upper left). ADR window was coupled to the FTS with the vacuum port. Spectral response of pixels in array A3 is shown on Figure 9 (upper right). Spectral response of pixels in array A5 is shown in lower left (TES_1 and TES_5) and in lower right (TES_16 and TES_7) of Figure 9. The spectra have been normalised to remove differential responsivity arising from the TES bias voltage and shunt resistors values as well as their array location with respect to the FTS incident beam. It is seen from the data that there are no out of band leaks in the spectral response.

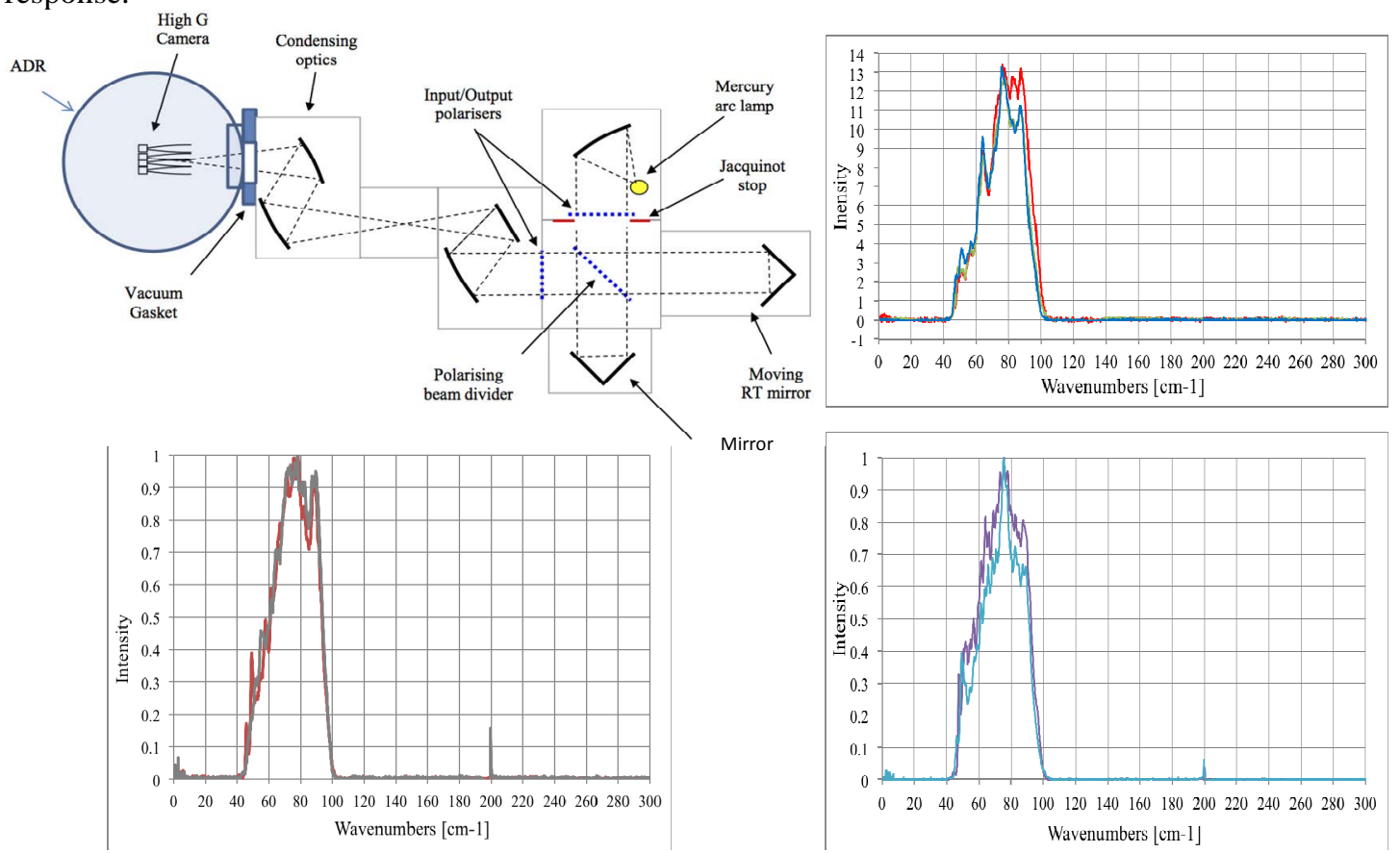

Figure 9: Upper left: schematics of FTS setup with ADR. Normalised spectral throughput of the TES pixels: upper right : TES_1 (red), TES_5 (green) and TES_10 (blue) of Array A3; lower left: TES_1 (grey), TES_5 (red) of Array A5; lower right: TES_7 (blue) and TES_16 (violet) of Array A5.

\subsection{Optical efficiency}

Optical efficiency of the camera pixel is the ratio of absorbed power at the TES to incident optical power at the horn entrance aperture, $\mathrm{P}_{\mathrm{abs}} / \mathrm{P}_{\text {opt }}$. We can determine $\mathrm{P}_{\mathrm{abs}}$ as the difference in $\mathrm{P}_{\text {TES }}$ with hot $(300 \mathrm{~K})$ and cold $(77 \mathrm{~K})$ blackbody source filling the camera beam. By alternately placing cold and hot loads in front of the window and measuring the IV curve we have determined the absorbed power in the TES pixel. To increase the accuracy of the data, we measured $P_{a b s}=P_{T E S}^{77 K}-P_{T E S}^{300 K}$ in the range of TES base temperatures. Left plot on Figure 10 shows $\mathrm{P}_{\mathrm{TES}}$ data with $300 \mathrm{~K}$ and 77K blackbody sources; the plot on the right is absorbed power by the TES. Figure 10 shows the representative data for pixel TES_1 in Array A5. 

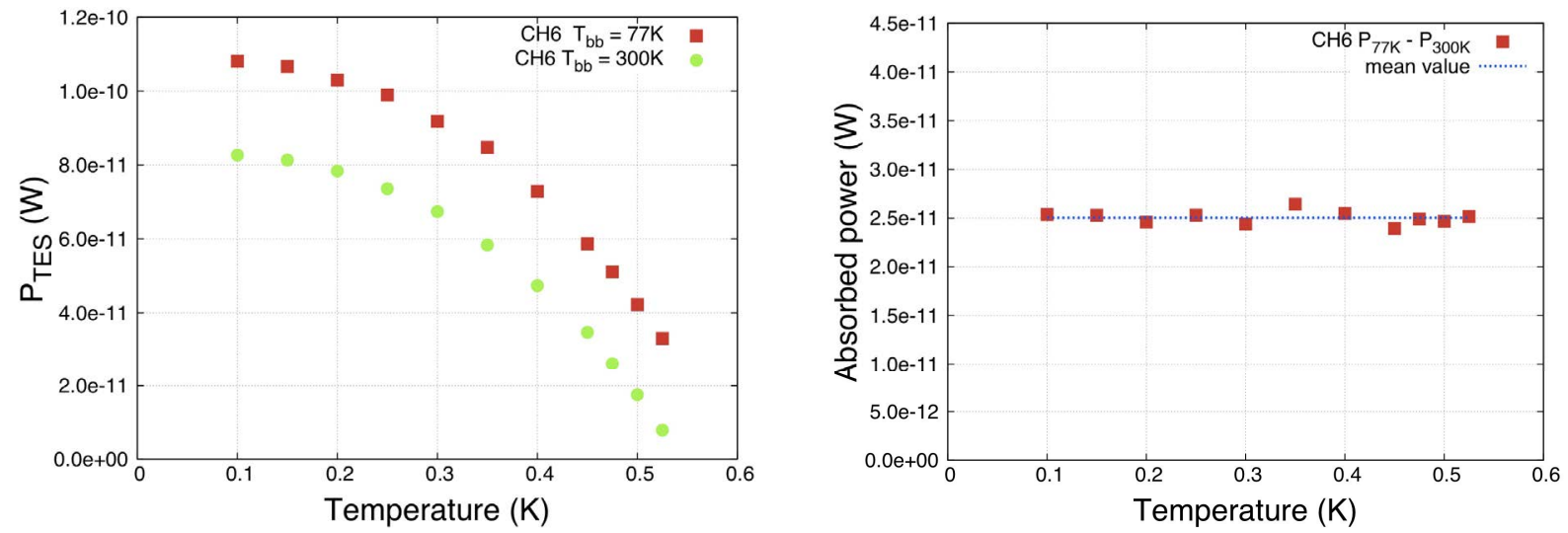

Figure 10: TES power as a function of the TES base temperature for TES_1 (array A5) is on the left. Red squares are the data for $77 \mathrm{~K}$ optical load and green circles show the data for $300 \mathrm{~K}$ optical load. Absorbed optical power for the same pixel is on the right.

The power incident at the horn aperture from a given beam filling source at temperature $T$ is given by:

$$
P_{L}=A \Omega \int_{0}^{\infty} \frac{2 f(v) h v^{3}}{c^{2}\left(e^{h v / k T}-1\right)} d v
$$

where $h$ is Planck's constant, $k$ is Boltzmann constant, $v$ is the frequency, $c$ is the speed of light and $f(v)$ is the filter transmission function. $f(v)$ is determined from the measured filter profiles multiplied together. This integral can be performed for both the black body source at room temperature $300 \mathrm{~K}$ and liquid nitrogen temperature $77 \mathrm{~K}$ to determine the power difference $\left(\mathrm{P}_{\mathrm{opt}}\right)$ at the horn aperture.

In determining the beam solid angle $(\Omega)$ we used a 1D cut across the antenna pattern and assumed it to be symmetrical (rotation about the pointing axis) to give the integrated solid angle. Knowing the geometrical horn area (A) of $2.1 \times 10^{-6}$ we determined an integrated solid angle of $6.92 \times 10^{-2}$ ster. The calculated optical power $\mathrm{P}_{\text {opt }}$, is $601.7 \mathrm{pW}$ (for sub-array A3) and $149.2 \mathrm{pW}$ (for sub-array A5). Note that the A5 sub-array measurements required an additional neutral density filter to prevent saturation.

Measured optical efficiency of TES pixels ranges from 14\% to $32 \%$ for the pixels with meshed absorbers in both subarrays A3 and A5. Pixels with filled absorbers on sub-array A5 are more efficient with efficiency values ranging from $34 \%$ to $63 \%$, where the highest number is comparable to the horn alone efficiency of $\sim 70 \%$. The difference in efficiencies of filled and meshed absorbers qualitatively was a result of using the same surface impedance as the filled absorbers. If the surface impedance was decreased to take account of the fill factor geometry an enhancement in the optical efficiency of $\sim 40 \%$ is expected. The optical efficiencies of the largest mesh absorber would then be in line with the filled absorbers.

\section{CONCLUSIONS}

We have reported on the design of the camera module for L-band and its optical characterization. Seven pixels from 2 arrays with filled and meshed absorbers were optically studied. Beam profiles of the pixels agree well with the model profiles and the spectral band of the camera is well defined. Optical efficiency ranges from $16 \%$ (meshed absorbers) to 63\% (filled absorbers). We found that the efficiency of filled absorbers is higher than meshed and $320 \mu \mathrm{m}$ absorbers are more efficient compared to $250 \mu \mathrm{m}$, which suggests that the position and distance of the absorber behind the exit aperture is critical.

Authors would like to acknowledge support from the European Space Agency through ESA-CTP contract 4000107657/13/NL/HB “Low Noise Equivalent Power TES Bolometers for FIR Applications”. 


\section{REFERENCES}

[1] P. Roelfsema, M. Giard, F. Najarro, K. Wafelbakker, W. Jellema, B.Jackson, B. Sibthorpe, M. Audard, Y. Doi, A. di Giorgio, M. Griffin, F. Helmich, I. Kamp, F. Kerschbaum, M. Meyer, D. Naylor, T. Onaka, A. Poglitch, L. Spinoglio, F. van der Tak, and B. Van-denbussche, Proc. SPIE 9143, 91431K (2014).

[2] B. D. Jackson, P. A. J. de Korte, J. van der Kuur, P. D. Mauskopf, J. Beyer, M. P.Bruijn, A. Cros, J. Gao, D. Griffin, R. den Hartog, M. Kiviranta, G. de Lange,B. van Leeuwen, C. Macculi, L. Ravera, N. Trappe, H. van Weers, and S. Withington,IEEE Transactions on Terahertz Science and Technology2, 12 (2012).

[3] D. J. Goldie, J. R. Gao, D. M. Glowacka, D. K. Griffin, R. Hijmering, P. Khosropanah,B. D. Jackson, P. D. Mauskopf, D. Morozov, J. A. Murphy, M. Ridder, N. Trappe,C. O’Sullivan, and S. Withington, Proc. SPIE 8452, 84520A (2012).

[4] D. Morozov, P. D. Mauskopf, P. Ade, M. Ridder, P. Khosropanah,M. Bruijn, J. van der Kuur, H. Hoevers, J.-R. Gao,and D. Griffin, IEEE Transactions on Applied Superconductivity21, 1 (2009).

[5] Audley, Michael D. and de Lange, Gert and Gao, Jian-Rong and Khosropanah, Pourya and Hijmering, Richard and Ridder, Marcel and Mauskopf, Philip D. and Morozov, Dmitry and Trappe, Neil A. and Doherty, Stephen, Review of Scientific Instruments, 87, 043103 (2016)

[6] PolyTech TMS-100 http://www.polytec.com/uk

[7] Thomas Keating Ltd. http://www.terahertz.co.uk

[8] D. J. Goldie, D. M. Glowacka, S. Withington, Jiajun Chen, P. A. R. Ade, D. Morozov, R. Sudiwala, N. A. Trappe, O. Quaranta, In these proceedings ().

[9] Physikalisch-Technische Bundesanstalt http://www.ptb.de

[10] Magnicon GmbH http://www.magnicon.com 